Draft version August 21, 2018

Preprint typeset using $\mathrm{IAT}_{\mathrm{E}} \mathrm{X}$ style emulateapj v. 5/2/11

\title{
X-RAY PHOTOIONIZED BUBBLE IN THE WIND OF VELA X-1 PULSAR SUPERGIANT COMPANION
}

\author{
JiŘí KRTiČKA ${ }^{1}$, JiŘí KubÁT ${ }^{2}$ AND JAN SKALICKÝ ${ }^{1}$ \\ ${ }^{1}$ Ústav teoretické fyziky a astrofyziky, Masarykova univerzita, Kotlářská 2, CZ-611 37 Brno, Czech Republic and \\ 2 Astromomický ústav Akademie věd České republiky, Fričova 298, CZ-251 65 Ondřejov, Czech Republic \\ Draft version August 21, 2018
}

\begin{abstract}
Vela X-1 is the archetype of high-mass X-ray binaries, composed of a neutron star and a massive $\mathrm{B}$ supergiant. The supergiant is a source of a strong radiatively-driven stellar wind. The neutron star sweeps up this wind, and creates a huge amount of X-rays as a result of energy release during the process of wind accretion. Here we provide detailed NLTE models of the Vela X-1 envelope. We study how the X-rays photoionize the wind and destroy the ions responsible for the wind acceleration. The resulting decrease of the radiative force explains the observed reduction of the wind terminal velocity in a direction to the neutron star. The X-rays create a distinct photoionized region around the neutron star filled with a stagnating flow. The existence of such photoionized bubbles is a general property of high-mass X-ray binaries. We unveiled a new principle governing these complex objects, according to which there is an upper limit to the X-ray luminosity the compact star can have without suspending the wind due to inefficient line driving.

Subject headings: stars: winds, outflows - stars: mass-loss - stars: early-type - hydrodynamics radiative transfer
\end{abstract}

\section{INTRODUCTION}

A high-mass X-ray binary (HMXB) is a binary star system consisting of a massive luminous hot star (frequently OB supergiant) and a compact object, either a neutron star or a black hole (Remillard \& McClintock 2006). A fraction of the stellar wind of the luminous hot star is trapped in the gravitational well of the compact object, and is accreted onto its surface (Davidson \& Ostriker 1973; Lamers et al. 1976). Part of the released potential energy of accreting material is transformed into $\mathrm{X}$ rays, resulting in one of the most powerful stellar X-ray sources. Such systems of stars in interaction belong to the most valuable astrophysical laboratories. The binary nature of the object enables us to determine stellar parameters precisely, which subsequently serve as a firm base for further study.

Vela X-1 (HD 77581, GP Vel) is the archetype of high-mass X-ray binaries, consisting of a neutron star and a massive B supergiant (Chodil et al. 1967; Brucato \& Kristian 1972; Barziv et al. 2001; Tomsick et al. 2010). The neutron star is a source of pulsed X-ray and $\gamma$-ray emission with a period of $283 \mathrm{~s}$ (McClintock et al. 1976; North et al. 1987), modulated both by the orbital motion and stochastic variations (Bildsten et al. 1997). The X-rays propagating through the hot star wind probe the wind structure, yielding information about the mass-loss rate and the velocity field (Watanabe et al. 2006). The perpetual X-ray variation (flaring) reveals the existence of some structure in the wind - clumping (Ducci et al. 2009; Fürst et al. 2010). On the other hand, X-rays also significantly influence the stellar wind, resulting in X-ray photoionization of its material (MacGregor \& Vitello 1982; Watanabe et al. 2006). Because the stellar wind of hot stars is mostly driven by the light absorption in the lines of heavier elements, the X-ray photoionization may influence the wind acceleration. Particularly, the appearance of highly charged ions, which absorb the light less effectively than low-charged ions, causes the decrease of the radiative force. Since this force is responsible for driving the wind, the wind flow may subsequently stagnate.

Numerical studies of stellar winds in HMXBs concentrate mainly on the multidimensional simulation of wind accretion (Blondin et al. 1990; Blondin \& Woo 1995; Feldmeier et al. 1996; Hadrava \& Cechura 2012), while the wind driving is simplified using force multipliers that take the X-ray irradiation into an account in an approximative way (Stevens \& Kallman 1990; Stevens 1991). This is a significant shortcoming, because the X-ray photoionization affects the radiative force, and consequently the amount and velocity of wind material accreted on the compact companion. Detailed modelling of ionization and excitation balance in the wind is crucial for the understanding of the influence of X-ray photoionization on the wind dynamics. The ionization and excitation balance should be properly derived using equations of statistical equilibrium 1 To remedy the situation, we provide wind models that include the influence of X-ray irradiation using up-to-date NLTE models.

\section{VELA X-1 PRIMARY WIND MODEL}

The applied models of the Vela $\mathrm{X}-1$ primary wind are based on the NLTE code with comoving frame (CMF) line force (Krtička \& Kubát 2010). Our models enable to selfconsistently predict wind structure just from the stellar parameters (the effective temperature, mass, radius, and chemical composition). Here we assume that the stellar wind of the supergiant component is symmetric with respect to the binary axis (connecting centers of both components) and that the stellar wind in the direction given by the inclination $\phi$ from the binary axis

\footnotetext{
1 This approach is usually referred to as non-LTE or NLTE and it means that the assumption of thermodynamic equilibrium is not used for evaluation of the excitation and ionization balance.
} 
can be locally described by a spherically symmetric wind model. The influence of the neutron star is taken into account by its inclusion as the source of external X-ray irradiation of the wind.

Basic parameters of Vela X-1 binary system are given in Table 1. Binary parameters and the physical parameters of binary members are taken from the spectroscopic analysis (van Kerkwijk et al. 1995). The effective temperature of the supergiant is taken from the tables of Straižys \& Kuriliene (1981) for the corresponding spectral type. The derived value relatively well agrees with the determination based on NLTE model atmospheres (Fraser et al. 2010). The parameters of the Xray source are adopted from observational analysis of Watanabe et al. (2006). For our calculations we assume the solar chemical composition (Asplund et al. 2009).

\subsection{Wind model without $X$-ray irradiation}

For supergiant parameters given in Table 1 we first calculated NLTE wind model with CMF line force neglecting the influence of the companion star. The emergent surface flux is taken from the $\mathrm{H}-\mathrm{He}$ spherically symmetric NLTE model stellar atmospheres (Kubát 2003). The predicted wind mass-loss rate $1.5 \times 10^{-6} \mathrm{M}_{\odot}$ year $^{-1}$ well agrees with its estimate derived from the observed $\mathrm{X}$ ray spectrum $1.5-2 \times 10^{-6} \mathrm{M}_{\odot}$ year $^{-1}$ Watanabe et al. 2006). The predicted terminal velocity $750 \mathrm{kms}^{-1}$ is lower than the observed one $1100 \mathrm{kms}^{-1}$ (Prinja et al. 1990). This disagreement may stem either from the model simplifications, inaccurate stellar parameters (e.g., metallicity), or from the sensitivity of the wind terminal velocity to a detailed ionization balance in the outer wind regions (Puls et al. 2000), which probably manifests itself in a significant scatter of the ratio of the terminal to the escape velocity for the stars with the same effective temperatures (e.g., Crowther et al. 2006).

The velocity structure of the wind model without Xray irradiation can be approximated by

$$
\begin{aligned}
\tilde{v}(r)=\left[v_{1}\left(1-\frac{R_{*}}{r}\right)+v_{3}\left(1-\frac{R_{*}}{r}\right)^{3}\right] \times \\
\left\{1-\exp \left[\gamma\left(\frac{r}{R_{*}}-1\right)^{2}\right]\right\},
\end{aligned}
$$

where

$$
v_{1}=1042 \mathrm{~km} \mathrm{~s}^{-1}, \quad v_{3}=-297 \mathrm{kms}^{-1}, \quad \gamma=-1220 .
$$

Note that the representation of the velocity law by a polynomial expansion provides better approximation than the ordinary " $\beta$-velocity law", because these polynomials may form a functional basis (Krtička \& Kubát 2011). The exponential term is included for a better fit of the velocity law close to the sonic point. The calculated X-ray opacity per unit mass averaged for radii $1.5 R_{*}-5 R_{*}$ may be approximated by

$$
\tilde{\kappa}^{\mathrm{X}}(\nu)= \begin{cases}a_{1}\left(\lambda-b_{1}\right)^{2}, & \lambda<\lambda_{1}, \\ a_{2}\left(\lambda+b_{2}\right)^{3}, & \lambda>\lambda_{1},\end{cases}
$$

where $\lambda=10^{8} \mathrm{c} / \nu, a_{1}=0.704 \mathrm{~g}^{-1} \mathrm{~cm}^{2}, b_{1}=1.056$, $a_{2}=4.06 \times 10^{-3} \mathrm{~g}^{-1} \mathrm{~cm}^{2}, b_{2}=11.41$, and $\lambda_{1}=20.18$.
We stress that $\lambda$ enters as a non-dimensional parameter here, which has for a convenience the same value as the wavelength in units of $\AA$.

\subsection{Modelling of the 2D wind structure}

The full treatment of the problem would essentially require a complex solution of $3 \mathrm{D}$ time-dependent hydrodynamic equations (Friend \& Castor 1982; Blondin et al. 1990; Feldmeier et al. 1996). However, because the typical wind flow-time $R_{*} / v_{\infty} \approx 0.3$ day is roughly by a factor of 30 shorter than the orbital period, we neglect the influence of the orbital motion on the wind structure. Consequently, we assume that the stellar wind of the supergiant component is axisymmetric with respect to the binary axis connecting centers of components. Moreover, we assume that the stellar wind in the direction given by the inclination $\phi$ from the binary axis can be modelled by a spherically symmetric wind (see Fig. 1). Consequently, in the orbital plane of the binary our $2 \mathrm{D}$ wind model consists of sectors of a circle.

The wind model in each sector is calculated using our NLTE wind code. Because the radial wind velocity may be non-monotonic in some sectors, we do not use the CMF line force for the calculation of these models directly, however the line radiative force is given by the force calculated in the Sobolev approximation (e.g., Castor 1974) multiplied by the ratio of the CMF to the Sobolev line force derived from the wind model that neglects the radiation from the companion star (Sect. 2.1).

The influence of the neutron star is taken into account only by inclusion of its X-ray radiation due to the wind accretion on the neutron star. This radiation irradiates the supergiant and interacts with its wind. To describe this effect, we add an additional term $J^{\mathrm{X}}(\nu, r, d)$ to the specific intensity $J(\nu, r, d)$ in the form

$$
J^{\mathrm{X}}(\nu, r, d)=\frac{L^{\mathrm{X}}(\nu)}{16 \pi^{2} d^{2}} \mathrm{e}^{-\tau(\nu, r, d)},
$$

where the optical depth along the given ray is ( $z$ measures the distance along this ray)

$$
\tau(\nu, r, d)=\int_{0}^{d} \kappa(\nu, z) \rho(z) \mathrm{d} z,
$$

$L^{\mathrm{X}}(\nu)$ is the X-ray luminosity per unit of frequency, $d$ is the distance from the given point in the supergiant wind region to the surface of the neutron star, $\kappa(\nu, z)$ is the absorption coefficient per unit of mass, and $\rho(z)$ is the wind density. The distribution of emergent X-rays $L^{\mathrm{X}}(\nu)$ is approximated by the power law (Watanabe et al. 2006). Energies higher than $3 \mathrm{keV}$, which are well above the ionization energies of all included ions were not considered in the model. The absorption coefficient and the density in Eq. (5) can be derived from models for individual sectors. However, to simplify our approach, for the calculation of $J^{\mathrm{X}}(\nu)$ we use fits following from Eqs. (11), (3)

$$
\begin{aligned}
\rho(z) & =\frac{\dot{M}}{4 \pi r^{2} v(z)}, \\
v(z) & =\min (\tilde{v}(r), \hat{v}(\phi)), \\
\kappa(\nu, z) & =\tilde{\kappa}^{\mathrm{X}}(\nu),
\end{aligned}
$$


Table 1

Parameters of Vela X-1 (HD 77581) binary system

\begin{tabular}{ccc}
\hline \hline & Value & Reference \\
\hline Parameter & Binary & \\
\hline Separation $D$ & $53.4 \mathrm{R}_{\odot}$ & van Kerkwiik et al. (1995) \\
Period $P$ & 8.96 day & van Kerkwiik et al. (1995) \\
\hline Spectral type & Supergiant & \\
Radius $R_{*}$ & B0.5Iae & \\
Mass $M$ & $30 \mathrm{R}_{\odot}$ & van Kerkwiik et al. $(1995)$ \\
Effective temperature $T_{\text {eff }}$ & $23.5 \mathrm{M}_{\odot}$ & van Kerkwiik et al. $(1995)$ \\
Wind mass-loss rate $\dot{M}$ & $27000 \mathrm{~K}$ & Straižys \& Kuriliene \\
Wind terminal velocity $v_{\infty}$ & $1.5 \times 10^{-6} \mathrm{M}_{\odot}$ year & this work \\
\hline & $750 \mathrm{~km} \mathrm{~s}^{-1}$ & this work \\
\hline Mass & Neutron star & \\
\hline X-ray luminosity $L_{\mathrm{X}}$ & $1.88 \mathrm{M}_{\odot}$ & van Kerkwiik et al. (1995) \\
\hline
\end{tabular}

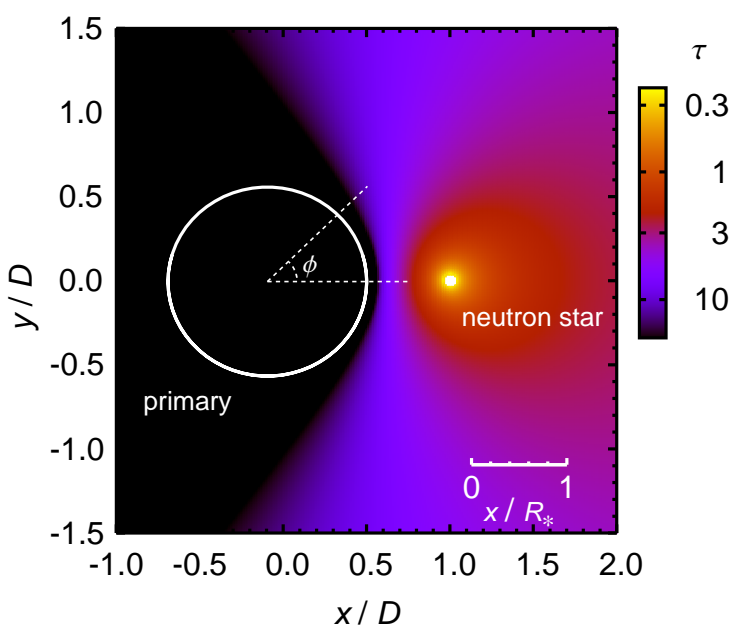

Figure 1. The optical depth for $830 \mathrm{eV}$ between the neutron star and a given point plotted in the plane containing the binary axis. The distances are given in the units of binary separation $D$ with a small ruler plotted in units of supergiant radius $R_{*}$. Note that the optical depth plotted in the figure is only an approximative one, because it was calculated using fits Eqs. (1), (3) to wind solution without any external X-ray irradiation. Anyway, the main trends are the same as in a more precise $2 \mathrm{D}$ model.

where the relation between the distance along the ray $z$ and the radius $r$ is derived from the geometry of the problem, and $\hat{v}(\phi)$ is an average velocity in the velocity plateau which occurs due to X-rays (see Fig. 3).

The models describing the wind in different sectors with inclination $\phi$ with respect to the binary axis are calculated for a sequence in $\phi$ with a step of $10^{\circ}$.

\section{WIND STRUCTURE IN 2D}

The X-ray source located on the surface of the neutron star influences the ionization state of the supergiant wind. The influence is stronger if the X-ray optical depth between a given point in the wind and the neutron star surface is lower. To illustrate this, in Fig. 1 we plot the optical depth (Eq. (5)) in a plane containing the binary axis. Due to the assumed symmetry of the problem, the
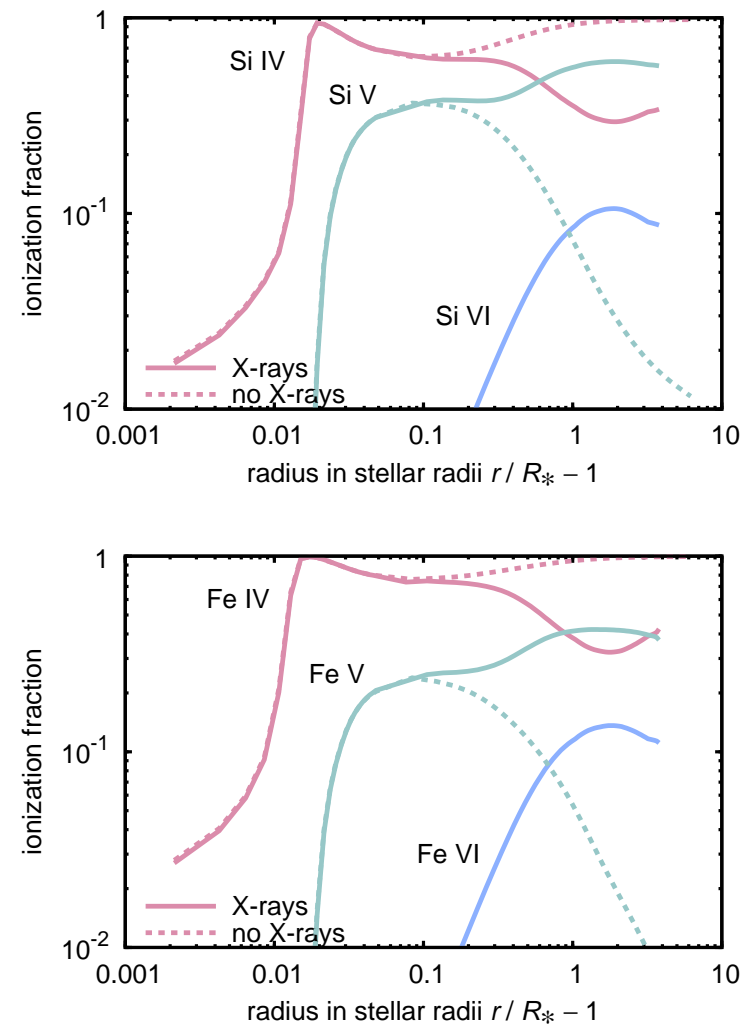

Figure 2. Influence of X-ray photoionization on the ionization state of selected elements. Calculated for a sector with $\phi=50^{\circ}$.

optical depth is axisymmetric with respect to the binary axis. The X-rays strongly penetrate the wind that directly faces the neutron star. On the other hand, due to geometrical reasons, the radial wind streams that are significantly inclined with respect to the neutron star are affected by $\mathrm{X}$-ray radiation at larger distances from the primary.

As a result of the X-ray photoionization of the wind, lower ionic states are effectively destroyed and higher ionic states appear in a nonnegligible amount. This can be seen from Fig. 2, where we compare the ionization fraction of selected ions in the model with and without $\mathrm{X}$-ray irradiation. The X-rays influence the wind ioniza- 


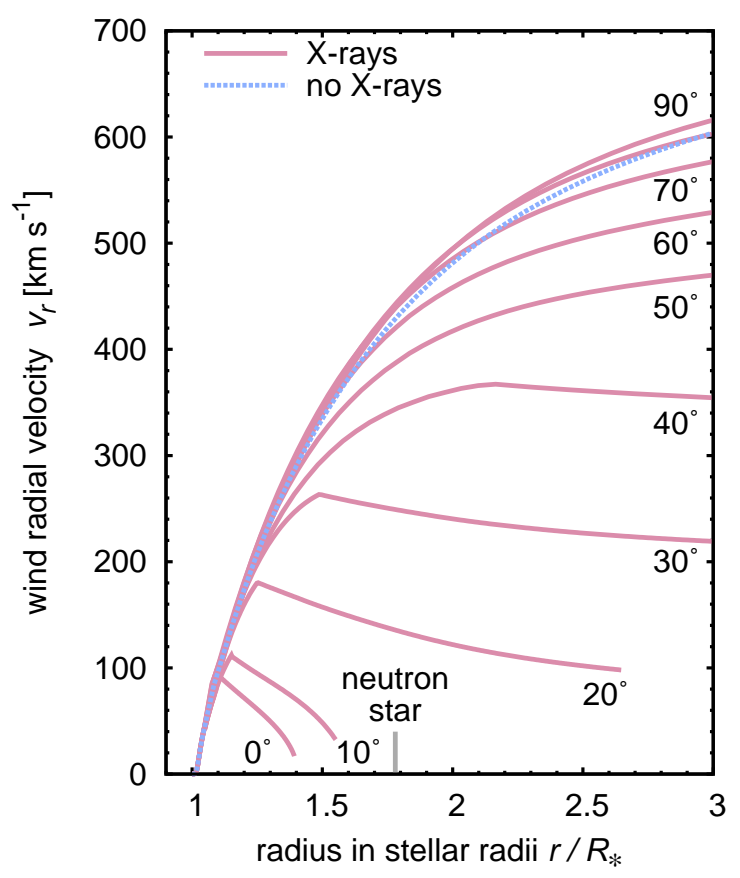

Figure 3. The radial wind velocity for different inclinations $\phi$ with respect to a neutron star. The position of the neutron star is marked in the graph.

tion state in the region where the X-ray optical depth between a given point and the neutron star is low, $\tau \lesssim 1$, i.e, to a distance comparable to an orbital separation $D$. $\mathrm{X}$-rays are not able to penetrate the wind close to the supergiant star (since $\tau \gg 1$ if we aim to approach the supergiant surface), consequently the ionization state of material there is not affected by X-rays.

Because the stellar wind of hot stars is accelerated by the light absorption in the lines of heavier element ions, any change of the wind ionization state affects the accelerating radiative force. On average, ions with higher charge are less effective in driving the wind, basically due to the lower number of their spectral lines. For a weak X-ray irradiation the emergence of new ionization states causes a slight increase of the radiative force (cf. Krtička \& Kubát 2009). On the other hand, for a strong irradiation when the degree of ionization is higher the radiative force significantly decreases (MacGregor \& Vitello 1982). Because this effect appears in the outer wind region and the wind mass-loss rate is determined in the inner wind region, only the wind velocity (see Fig. 3), and not the wind mass-loss rate (MacGregor \& Vitello 1982) is affected by $\mathrm{X}$-ray photoionization. This is especially apparent for lower wind inclinations $\left(\phi<40^{\circ}\right)$, where the wind radial velocity becomes nonmonotonic. The decrease in the radiative force is so strong there that the accelerating wind solution is not possible any more, and the wind velocity switches to decelerating overloaded solutions (Feldmeier \& Shlosman 2000) with a typical kink in the velocity profile. This has further consequences for even lower wind inclinations. The radiative force in the rays that closely pass the neutron star $\left(\phi<15^{\circ}\right)$ is so strongly affected by the X-ray photoionization that the radiatively driven wind in these directions never reaches the neutron star.

As the result of X-ray photoionization, a region (bubble) around the neutron star is created, in which the wind stagnates at low velocities, and consequently high densities. These results explain the detection of lowspeed wind regions in the direction of the neutron star by Watanabe et al. (2006).

\section{DISCUSSION}

\subsection{X-ray luminosity}

Part of the mass which leaves the primary star via its wind can be accreted onto the compact component. Its potential energy is then released in the form of X-ray radiation. Let us adopt an approximative Hoyle-Lyttleton treatment to estimate the X-ray luminosity. For moving medium, the radius from which the matter can be accreted onto a point mass is given by the relation $r_{\mathrm{HL}}=$ $2 G M_{\mathrm{X}} / v^{2}$, where $M_{\mathrm{X}}$ is the mass of the compact component (neutron star), $v^{2}=v_{\text {wind }}^{2}+v_{\text {orb }}^{2}, v_{\text {orb }}$ is the orbital velocity of the compact component, and $v_{\text {wind }}$ is the wind velocity at the distance of the compact component (denoted by $D$ ). Given $v_{\text {orb }}=300 \mathrm{~km} \mathrm{~s}^{-1}$ and our simulated wind structure, we find that the neutron star is able to collect matter only from a narrow cone defined by the value of $\phi<15^{\circ}$ (for $\phi=15^{\circ}$ and the distance $D$ we have $v_{\text {wind }}=32.0 \mathrm{~km} \mathrm{~s}^{-1}$ and $\left.r_{\mathrm{HL}}=0.17 D\right)$. We can express the relation between the accretion rate $\dot{M}_{\text {acc }}$ and the mass loss rate $\dot{M}$ from the supergiant as (Watanabe et al. 2006) $\dot{M}_{\mathrm{acc}}=\dot{M} r_{\mathrm{HL}}^{2} /\left(4 D^{2}\right)$. Then the X-ray luminosity $L_{\mathrm{X}}=G M_{\mathrm{X}} \dot{M}_{\mathrm{acc}} / R_{\mathrm{X}}$ is $L_{\mathrm{x}}=8.8 \times 10^{37} \mathrm{erg} \mathrm{s}^{-1}$. This value is approximately one order of magnitude higher than the observed one (Watanabe et al. 2006). The reason of this difference is the fact that only a small fraction of matter in the accretion cone gets finally accreted and the rest falls back to the surface of the primary as a consequence of the flow stagnation between the supergiant and the neutron star.

\subsection{Existence of two types of solutions}

The wind equations allow the existence of two types of solutions giving different X-ray luminosities and wind velocities. The solution presented here appears in the case of strong X-ray source, which significantly affects the wind ionization state and consequently also the radiative force. This results in a slow wind that can be accreted by the neutron star in large amounts producing a strong $\mathrm{X}$-ray source.

Another type of solution may occur in the case of a weak X-ray source that does not significantly influence the wind ionization state. The radiation force becomes higher, similar to that without any X-ray irradiation. This results in faster outflow roughly corresponding to the "no X-rays" case in Fig. 3. Due to the dependence of the accretion rate on the velocity via $\dot{M}_{\text {acc }} \sim v^{-4}$ and having roughly two times higher $v$, the X-ray luminosity is by an order of magnitude lower in this case (since $L_{\mathrm{X}} \sim$ $\left.\dot{M}_{\text {acc }}\right)$. This agrees with adopted assumption of weak Xray source. Consequently, also this is a possible solution of wind equations.

Different types of solutions may appear in different systems, or even an external perturbation may cause switching between these two wind solutions in a particular bi- 


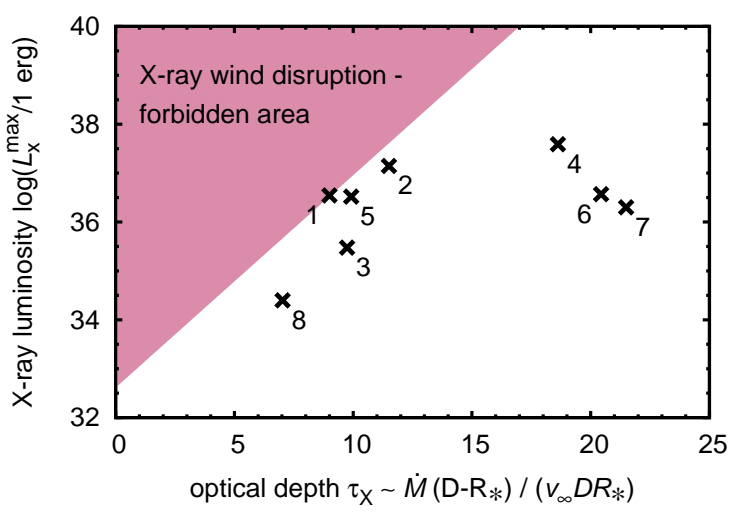

Figure 4. The location of forbidden X-ray luminosities as a function of the optical depth parameter (Eqs. (9), (10). Observed parameters of individual HMXBs (crosses) are located outside the highlighted forbidden area. Here we plotted points (denoted by numbers) for binaries Vela X-1 (1, Watanabe et al. 2006), Cyg X-1 (2, Yan et al.|2008), IGR J19140+0951 (3, Prat et al. 2008), Wray 977 (4, Kaper et al.|2006), 4U 2206+54 (5, Ribó et al.|2006), 4U 0114+65 (6, Reig et al. 1996; Mukheriee \& Paul 2006), 4U 1907+09 (7, Cox et al.|2005), and LS 5039 (8, Casares et al.|2005). The plot of the maximum luminosity was derived using the optical depth between the neutron star and the wind critical point $\tau_{\mathrm{X}}($ Vela X-1) $=9$.

nary system, possibly contributing to the variability of X-ray luminosity. This variability might be accompanied by the variability of the distribution of emitted $\mathrm{X}$ rays, if the accretion regime changes with accretion rate (Shakura et al. 2012).

\subsection{Implication for other $H M X B s$}

For a slightly lower mass-loss rate or for a slightly higher X-ray luminosity than assumed here the X-rays could penetrate deeply into the stellar wind and significantly influence the ionization state at the wind base resulting in the decrease of the radiative force. This could lead to the disruption of the stellar wind and a significant decrease of X-ray luminosity, possibly providing another contribution to the overall X-ray variability observed in Vela X-1 (Kreykenbohm et al. 2008).

This means that there exists a maximum X-ray luminosity the compact star can have for a given geometry of the system and wind mass-loss rate. Assuming that the Vela X-1 luminosity is the maximum one, the maximum allowed X-ray luminosities for other HMXBs $L_{\mathrm{X}}^{\max }$ can be derived using the optical depth (compare with the ionization parameter $\xi$, Tarter et al. 1969) $\tau_{\mathrm{X}}=\int_{R_{*}}^{D} \kappa \rho \mathrm{d} r \sim$ $\dot{M}\left(D-R_{*}\right) /\left(v_{\infty} D R_{*}\right)$ and corresponding parameters of Vela X-1 as

$$
L_{\mathrm{X}}^{\max } e^{-\tau_{\mathrm{X}}}=L_{\mathrm{X}}\left(\text { Vela X-1) } e^{-\tau_{\mathrm{X}}(\text { Vela X-1)}},\right.
$$

or, in scaled quantities

$$
\begin{aligned}
\log \left(L_{\mathrm{X}}^{\max } / 1 \mathrm{erg}\right)=32.6+3.9\left(\frac{\dot{M}}{1.5 \times 10^{-6} \mathrm{M}_{\odot} \text { year }^{-1}}\right) \\
\quad \times\left(\frac{v_{\infty}}{750 \mathrm{~km} \mathrm{~s}^{-1}}\right)^{-1}\left(\frac{\left(D R_{*}\right) /\left(D-R_{*}\right)}{68.5 \mathrm{R}_{\odot}}\right)^{-1} \cdot(10)
\end{aligned}
$$

Here $\dot{M}, v_{\infty}, R_{*}$, and $D$ are the wind mass-loss rate, the terminal velocity, radius of luminous component, and the binary separation for individual HMXBs.
The results of observations for other HMXBs collected in Fig. 4 clearly support the picture that there exists a maximum allowed X-ray luminosity, which depends on the wind and geometry parameters. Some systems lie close to the boundary of the forbidden area, whereas others have typically higher wind mass-loss rate $\dot{M} \gtrsim$ $5 \times 10^{-6} \mathrm{M}_{\odot}$ year $^{-1}$ that keeps them out of the boundary. The position of individual stars in this diagram may vary with time due to the existence of two possible solutions of wind equations (as discussed in Sect. 4.2).

\subsection{Mass-loss rate determination}

Our results support the mass-loss rate predictions based on up-to-date wind models in two ways. First, our mass-loss rate prediction of HD77581, $1.5 \times 10^{-6} \mathrm{M}_{\odot}$ year $^{-1}$, agrees with the value estimated from X-ray spectroscopy, $1.5-2 \times 10^{-6} \mathrm{M}_{\odot}$ year $^{-1}$ (Watanabe et al. 2006). Moreover the wind mass-loss rate cannot be lower than this value, because a decrease of the wind mass-loss rate would lead to lower X-ray opacity, stronger wind X-ray photoionization close to the star, and even more significant reduction of the radiative force. This would finally cause a disruption of the wind and a disappearance of X-ray emission. This imposes a strong lower limit on the observational wind mass-loss rate estimates, that is in agreement with current massloss rate predictions (Vink et al. 2001; Krtička \& Kubát 2010).

\subsection{Limitations of the present models}

The consistent inclusion of the X-ray irradiation, which is an advantage of our models, also determines their shortcomings. The coupled solution of NLTE and radiative transfer equations is significantly time-consuming even in 1D. Its inclusion into multidimensional timedependent simulations is likely beyond the possibilities of current computers. Consequently, while one part of the problem solution is treated in detail, the second one is simplified. The correct picture of the flow in the HMXBs should take into an account the results of both approaches: the stagnation of the flow in the direction towards the neutron star, which is studied in this paper, and a complex 2D picture of the wind accretion on the neutron star (Blondin et al. 1990; Blondin \& Woo 1995; Feldmeier et al. 1996).

On the other hand, there are effects that are not described by any of the available models. It is well established that the hot star wind is inhomogeneous on small scales (clumped, see Hamann et al. 2008). In the case of HMXBs, clumping (which favors recombination) may affect the region in which the photoionized bubble is formed (Oskinova et al. 2012). However, the hydrodynamical simulations (Feldmeier et al. 1997; Runacres \& Owocki 2002) predict that clumping starts above the critical point of the wind solution, consequently, it does not affect wind mass-loss rates and terminal velocities. Thus we expect that clumping does not significantly influence the results of our models.

The wind inhomogeneities likely cause the high variability of the X-ray source (Fürst et al. 2010; Oskinova et al. 2012). The calculation of the wind ionization should in fact account for such time-dependent Xray photoionization. However, because the typical flow 
time of the wind is longer than the typical timescale of $\mathrm{X}$-ray variability, we expect that our models are able to reproduce the mean effect of the X-ray photoionization. On the other hand, the variable X-ray ionization source causes temporal changes of the radiative acceleration, providing external perturbation which may contribute to the natural wind clumping. As a result, the primary wind may be more clumpy than the wind of a similar single supergiant.

\section{CONCLUSIONS}

We provide detailed numerical models of the influence of X-rays on the supergiant wind in the Vela X-1 binary system. The effect of X-ray photoionization on the radiative force and wind dynamics has never been studied using appropriate NLTE wind models. The X-rays photoionize the wind and destroy the ions responsible for the wind acceleration. This results in flow stagnation in the vicinity of the neutron star, which was identified in observations. For a sufficiently strong X-ray source the wind that directly faces the neutron star falls back on the mass losing star and never reaches the compact companion.

We have shown that there is an upper limit to the X-ray luminosity the compact star can have without disrupting the stellar wind. For a higher luminosity than the limiting one the decrease of the wind acceleration is so strong that no wind material would reach the neutron star. This theoretical picture of the maximum X-ray luminosity is supported by observation of many high-mass $\mathrm{X}$-ray binaries.

The wind equations allow the existence of two types of solutions with different X-ray luminosities and wind velocities. The case of a strong X-ray source, which significantly affects the wind ionization, leads to accretion of slow wind in large amounts resulting in a strong $\mathrm{X}$ ray source. On the other hand, a weak X-ray source that does not significantly influence the wind ionization results in accretion of fast wind in low amounts producing a weak X-ray source. Different types of solution may appear in different binary systems, or perturbations may cause switching between these two types of wind solutions contributing to the X-ray variability.

The predicted mass-loss rate agrees with the value estimated from X-ray spectroscopy. Moreover, the wind mass-loss rate cannot be lower than this value, because a decrease of the wind mass-loss rate would lead to the disruption of the wind and disappearance of the X-ray emission. This supports the reliability of current massloss rate predictions.

We thank an anonymous referee for valuable comments on the manuscript. This work was supported by grants GA ČR 205/08/0003 and MUNI/A/0968/2009.

\section{REFERENCES}

Asplund, M., Grevesse, N., Sauval, A. J., \& Scott, P. 2009, ARA\&A, 47, 481
Barziv, O. Kaper, L., Van Kerkwijk, M. H., Telting, J. H., Van Paradijs, J. 2001, A\&A, 377, 925

Bildsten, L., Chakrabarty, D., Chiu, J. et al. 1997 ApJS, 113, 367

Blondin, J. M., Woo, J. W. 1995, ApJ, 445, 889

Blondin, J. M., Kallman, T. R., Fryxell, B. A., \& Taam, R. E. 1990, ApJ, 356, 591

Brucato, R. J., \& Kristian, J. 1972, ApJ, 173, L105

Castor J. I., 1974, MNRAS, 169, 279

Casares, J., Ribó, M., Ribas, I. et al.2005 MNRAS, 364, 899

Chodil, G., Mark, H., Rodrigues, R., Seward, F. D., \& Swift, C. D. 1967, ApJ, 150, 57

Cox, N. L. J., Kaper, L., \& Mokiem, M. R. 2005, A\&A, 436, 661

Crowther, P. A., Lennon, D. J., \& Walborn, N. R. 2006, A\&A, 446,279

Davidson, K., \& Ostriker, J. P. 1973, ApJ, 179, 585

Ducci, L., Sidoli, L., Mereghetti, S., Paizis, A., \& Romano, P. 2009, MNRAS, 398, 2152

Feldmeier, A., \& Shlosman, I. 2000, ApJ, 532, L125

Feldmeier, A., Anzer, U., Boerner, G., \& Nagase, F. 1996, A\&A, 311,793

Feldmeier A., Puls J., Pauldrach A. W. A., 1997, A\&A, 322, 878

Fraser, M., Dufton, P. L., Hunter, I., \& Ryans, R. S. I. 2010, MNRAS, 404, 1306

Friend, D. B., \& Castor, J. I. 1982, ApJ, 261, 293

Fürst, F., Kreykenbohm, I., Pottschmidt, K. et al. 2010, A\&A, 519, A37

Hadrava, P., \& Čechura, J. 2012, A\&A, 542, A42

Hamann W.-R., Feldmeier A., Oskinova L. (eds.), 2008, Clumping in Hot Star Winds, Universitätsverlag Potsdam, Potsdam

Kaper, L., van der Meer, A., \& Najarro, F. 2006, A\&A, 457, 595

Kreykenbohm, I., Wilms, J., Kretschmar, P. et al. 2008, A\&A, 492,511

Krtička, J., \& Kubát, J. 2009, MNRAS, 394, 2065

Krtička, J., \& Kubát, J. 2010, A\&A, 519, A50

Krtička, J., \& Kubát, J. 2011, A\&A, 534, A97

Kubát, J., in Modelling of Stellar Atmospheres, N. E. Piskunov,

W. W. Weiss, D. F. Gray, Eds. IAU Symp. 210, (ASP Conf. Ser, San Francisco, 2003), p. A8

Lamers, H. J. G. L. M., van den Heuvel, E. P. J., \& Petterson, J. A. 1976, A\&A, 49, 327

MacGregor, K. B., \& Vitello, P. A. J. 1982, ApJ, 259, 267

McClintock, J. E., Rappaport, S., Joss, P. C. et al. 1976, ApJ, 206, L99

Mukherjee, U., \& Paul, B. 2006, A\&A, 27, 37

North, A. R., Raubenheimer, B. C., de Jager, O. C., van Tonder, A. J., van Urk, G. 1987, Nature, 326, 567

Oskinova, L. M., Feldmeier, A., Kretschmar, P. 2012, MNRAS, in press (arXiv:1201.1915)

Prat, L., Rodriguez, J., Hannikainen, D. C., \& Shaw, S. E. 2008, MNRAS, 389, 301

Prinja, R. K., Barlow, M. J., \& Howarth, I. D. 1990, ApJ, 361, 607

Puls, J., Springmann, U., \& Lennon, M. 2000, A\&AS, 141, 23

Remillard, R. A., \& McClintock, J. E. 2006, ARA\&A 44, 49

Ribó, M., Negueruela, I., Blay, P., Torrejón, J. M., \& Reig, P. 2006, A\&A, 449, 687

Reig, P., Chakrabarty, D., Coe, M. J. et al. 1996, A\&A, 311, 879

Runacres, M. C, Owocki, S. P. 2002, A\&A 381, 1015

Shakura, N., Postnov, K., Kochetkova, A., \& Hjalmarsdotter, L. 2012, MNRAS, 420, 216

Stevens, I. R. 1991, ApJ, 379, 310

Stevens, I. R., Kallman, T. R. 1990, ApJ, 365, 321

Straižys, V., \& Kuriliene, G. 1981, Ap\&SS, 80, 353

Tarter, C. B., Tucker, W. H.; Salpeter, E. E. 1969, ApJ, 156, 943

Tomsick, J. A., \& Muterspaugh, M. W. 2010, ApJ, 719, 958

van Kerkwijk, M. H., van Paradijs, J., \& Zuiderwijk, E. J., et al. 1995, A\&A, 303, 483

Vink, J. S., de Koter, A., \& Lamers, H. J. G. L. M. 2001, A\&A, 369,574

Watanabe, S., Sako, M., Ishida, M. et al. 2006, ApJ, 651, 421

Yan, J., Liu, Q., \& Hadrava, P. 2008, AJ, 136, 631 